\title{
Effect of nasal balloon autoinflation in children with otitis media with effusion in primary care: an open randomized controlled trial
}

\author{
Ian Williamson MD, Jane Vennik MRes, Anthony Harnden MBChB MSc, Merryn Voysey MBiostat, \\ Rafael Perera DPhil, Sadie Kelly PhD, Guiqing Yao PhD, James Raftery PhD, David Mant MBChB MA, \\ Paul Little MD
}

CMAJ Podcasts: author interview at soundcloud.com/cmajpodcasts/141608-res

See also page 949 and www.cmaj.ca/lookup/doi/10.1503/cmaj.150527

\begin{abstract}
Background: Otitis media with effusion is a common problem that lacks an evidence-based nonsurgical treatment option. We assessed the clinical effectiveness of treatment with a nasal balloon device in a primary care setting.
\end{abstract}

\begin{abstract}
Methods: We conducted an open, pragmatic randomized controlled trial set in 43 family practices in the United Kingdom. Children aged 4-11 years with a recent history of ear symptoms and otitis media with effusion in 1 or both ears, confirmed by tympanometry, were allocated to receive either autoinflation 3 times daily for 1-3 months plus usual care or usual care alone. Clearance of middle-ear fluid at 1 and 3 months was assessed by experts masked to allocation.
\end{abstract}

Results: Of 320 children enrolled, those receiving autoinflation were more likely than controls to have normal tympanograms at 1 month
(47.3\% [62/131] v. 35.6\% [47/132]; adjusted relative risk [RR] 1.36, 95\% confidence interval $[\mathrm{Cl}]$ 0.99 to 1.88$)$ and at 3 months $(49.6 \%$ [62/125] v. $38.3 \%$ [46/120]; adjusted RR 1.37, 95\% Cl 1.03 to 1.83 ; number needed to treat $=9$ ). Autoinflation produced greater improvements in earrelated quality of life (adjusted between-group difference in change from baseline in OMQ-14 [an ear-related measure of quality of life] score $-0.42,95 \% \mathrm{Cl}-0.63$ to -0.22 ). Compliance was $89 \%$ at 1 month and $80 \%$ at 3 months. Adverse events were mild, infrequent and comparable between groups.

Interpretation: Autoinflation in children aged 4-11 years with otitis media with effusion is feasible in primary care and effective both in clearing effusions and improving symptoms and ear-related child and parent quality of life. Trial registration: ISRCTN, No. 55208702.
$\mathrm{O}$ titis media with effusion, also known as glue ear, is an accumulation of fluid in the middle ear, without symptoms or signs of an acute ear infection. It is often associated with viral infection. ${ }^{1-3}$ The prevalence rises to $46 \%$ in children aged $4-5$ years, ${ }^{4}$ when hearing difficulty, other ear-related symptoms and broader developmental concerns often bring the condition to medical attention. ${ }^{3,5,6}$ Middle-ear fluid is associated with conductive hearing losses of about 15-45 dB HL. ${ }^{7}$ Resolution is clinically unpredictable, ${ }^{8-10}$ with about a third of cases showing recurrence. ${ }^{11}$ In the United Kingdom, about 200000 children with the condition are seen annually in primary care. ${ }^{12,13}$ Research suggests some children seen in primary care are as badly affected as those seen in hospital. ${ }^{7,9,14,15}$ In the United States, there were 2.2 million diagnosed episodes in 2004, costing an estimated $\$ 4.0$ billion. ${ }^{16}$ Rates of ventilation tube surgery show variability between countries, ${ }^{17-19}$ with a declining trend in the UK. ${ }^{20}$

Initial clinical management consists of reasonable temporizing or delay before considering surgery. ${ }^{13}$ Unfortunately, all available medical treatments for otitis media with effusion such as antibiotics, antihistamines, decongestants and intranasal steroids are ineffective and have unwanted effects, and therefore cannot be recommended. ${ }^{21-23}$ Not only are antibiotics ineffective, but resistance to them poses a major threat to public health. ${ }^{24,25}$ Although surgery is effective for a carefully selected minority, ${ }^{13,26,27}$ a simple low-cost, nonsurgical treatment option could ben-
Competing interests: None declared.

This article has been peer reviewed.

Accepted: June 10, 2015

Online: July 27, 2015

Correspondence to:

Ian Williamson,

igw@soton.ac.uk

CMAJ 2015. DOI:10.1503/ cmaj.141608 
efit a much larger group of symptomatic children, with the purpose of addressing legitimate clinical concerns without incurring excessive delays.

Autoinflation using a nasal balloon device is a low-cost intervention with the potential to be used more widely in primary care, but current evidence of its effectiveness is limited to several small hospital-based trials ${ }^{28}$ that found a higher rate of tympanometric resolution of ear fluid at 1 month. ${ }^{29-31}$ Evidence of feasibility and effectiveness of autoinflation to inform wider clinical use is lacking. ${ }^{13,28}$ Thus we report here the findings of a large pragmatic trial of the clinical effectiveness of nasal balloon autoinflation in a spectrum of children with clinically confirmed otitis media with effusion identified from primary care.

\section{Methods}

\section{Study design and participants}

We carried out an open, pragmatic randomized controlled trial in primary care. We examined the difference in effectiveness between autoinflation 3 times daily for 1-3 months plus usual care and usual care alone.

The study was piloted in 4 practices. The main study recruited children from 43 general practices from 17 primary care trusts (independent local groups) in the UK, between January 2012 and February 2013. Most (89\%) children were identified by practice-based computer search, and the rest were recruited through opportunistic case finding by practitioners, nurses and health visitors. Tympanometry and recruitment were undertaken by practice-based nurses. The study protocol is available in Appendix 1 at www.cmaj.ca/lookup/suppl/doi:10.1503/cmaj. 141608/-/DC1.

The National Research Ethics Service gave ethics approval for this study. The 17 participat- ing primary care trusts gave National Health Service approval.

\section{Eligibility criteria}

Children were eligible for inclusion if they were attending school and aged 4-11 years (deemed an age likely to be able to comply with autoinflation); had a history of hearing loss or other relevant ear-related problems in the previous 3 months; and had objective otoscopic and tympanometric confirmation of otitis media with effusion in at least 1 ear (i.e., had 1 or 2 type-B tympanograms using a modified Jerger classification) at the point of randomization (Table 1)..$^{32,33}$ Children were excluded if they had current clinical features of acute otitis media (e.g., ear pain, fever or otoscopic features of acute inflammation), recent or planned ear surgery, a known latex allergy or a recent nosebleed.

\section{Randomization and masking}

An independent external agency provided a centralized Web-based computer-generated randomization system (www.sealedenvelope.com) for nurses to access while recruiting children. The Oxford Primary Care Clinical Trials Unit independently managed, coordinated, analyzed and checked the data validity. The randomization involved an algorithm with minimization based on 3 variables: age, sex and baseline severity (bilateral v. unilateral type-B tympanograms). ${ }^{9}$ Because of the nature of the intervention, use of placebo was not possible, and therefore nurses, children and families were not masked to treatment allocation.

\section{Procedures}

All participating parents and children received information sheets, and parents gave written informed consent for screening to the research nurse. Children were invited to give written assent.

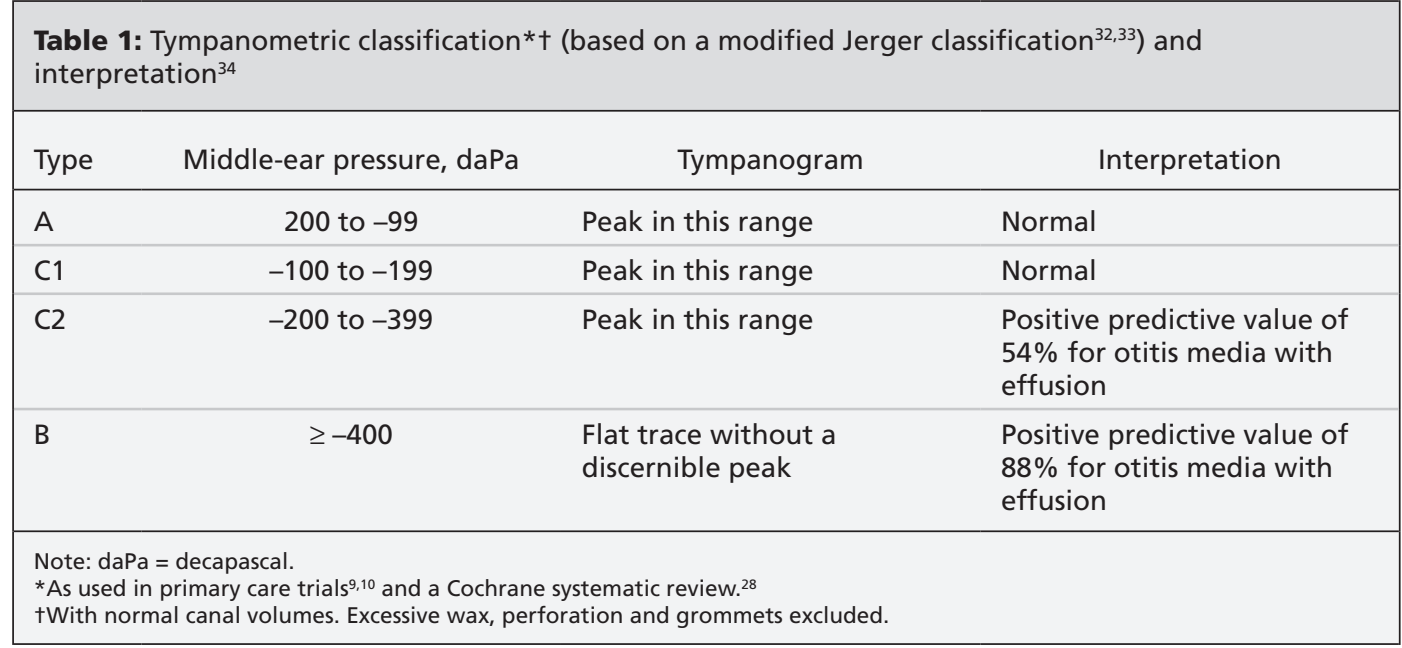


The simple autoinflation method involved inflating a purpose-manufactured balloon (Otovent) by blowing through each nostril into a connecting nozzle ${ }^{31}$ (Figure 1). Children receiving treatment were instructed by watching the nurse or parent demonstrate the procedure after stretching the balloon. The schedule involved inflating the balloon 3 times daily for an initial period of 1 month. Children still recording a type-B tympanogram in either ear at 1 month were advised to continue with autoinflation for a further 2 months. At the end of the study, affected children in the arm receiving usual care were offered a 1-month treatment pack.

All nurses received training in the study methods, including tympanometry and interpretation (from an audiologist), updates on otoscopy and ways of maximizing study compliance. Hand-held calibrated MTP10 tympanometers (with printout facilities) were used.

\section{Study outcomes}

We assessed outcomes at 1 and 3 months after randomization, during which time natural resolution effects would be expected to occur for some children. ${ }^{7,9}$

Our main outcome was the difference between groups in the proportion of children showing definite tympanometric resolution (i.e., normal middle-ear pressure, defined by a type- $\mathrm{A}$ or $-\mathrm{C} 1$ tympanogram) in at least 1 affected ear per child at 1 and 3 months. Intermediate type-C2 tympanograms showing negative pressure were considered insufficiently stringent for resolution of fluid (Table 1). ${ }^{32,34}$ We chose tympanometric outcomes because they allowed blinding, have been well validated previously, ${ }^{2,34}$ are regarded as a good choice for primary care studies ${ }^{13,35}$ and are used in meta-analysis. ${ }^{28}$ Two members of the trial team, trained in tympanometry and masked to allocation, independently reviewed anonymized tympanometry printouts. The expert interrater agreement was $89 \%$, and disagreement was settled by a third independent audiologist. We found a Cohen $\kappa$ of 0.7 for the level of agreement between nurses and masked experts.

Ear-related quality of life was measured at 3 months using the OMQ-14, an instrument developed by a process of refinement and iteration from large clinical and trial datasets of otitis media with effusion that optimized item mapping onto the Health Utilities Index. ${ }^{7,9,15,36}$ In addition, parents completed weekly diaries to record symptoms, adverse events and compliance. Days during which parents reported their child had hearing loss, earache, days off school, days requiring pain relief and sleep disturbance were summarized as days with any problem. We did not conduct pure tone audiometry because it cannot be done with adequate precision in nonspecialist settings.

\section{Sample size}

A total sample of 295 children was required to provide $90 \%$ power $(5 \% \alpha)$ to detect a treatment effect (odds ratio [OR]) of 2.4, and allowing for $15 \%$ loss to follow-up at 1 month. ${ }^{28}$ The study was also powered to detect a clinically important difference of 0.3 of a standard deviation (SD) in total OMQ-14 score.

\section{Statistical analysis}

We performed a modified intention-to-treat analysis, ignoring reported compliance but excluding children for whom no outcome mea-

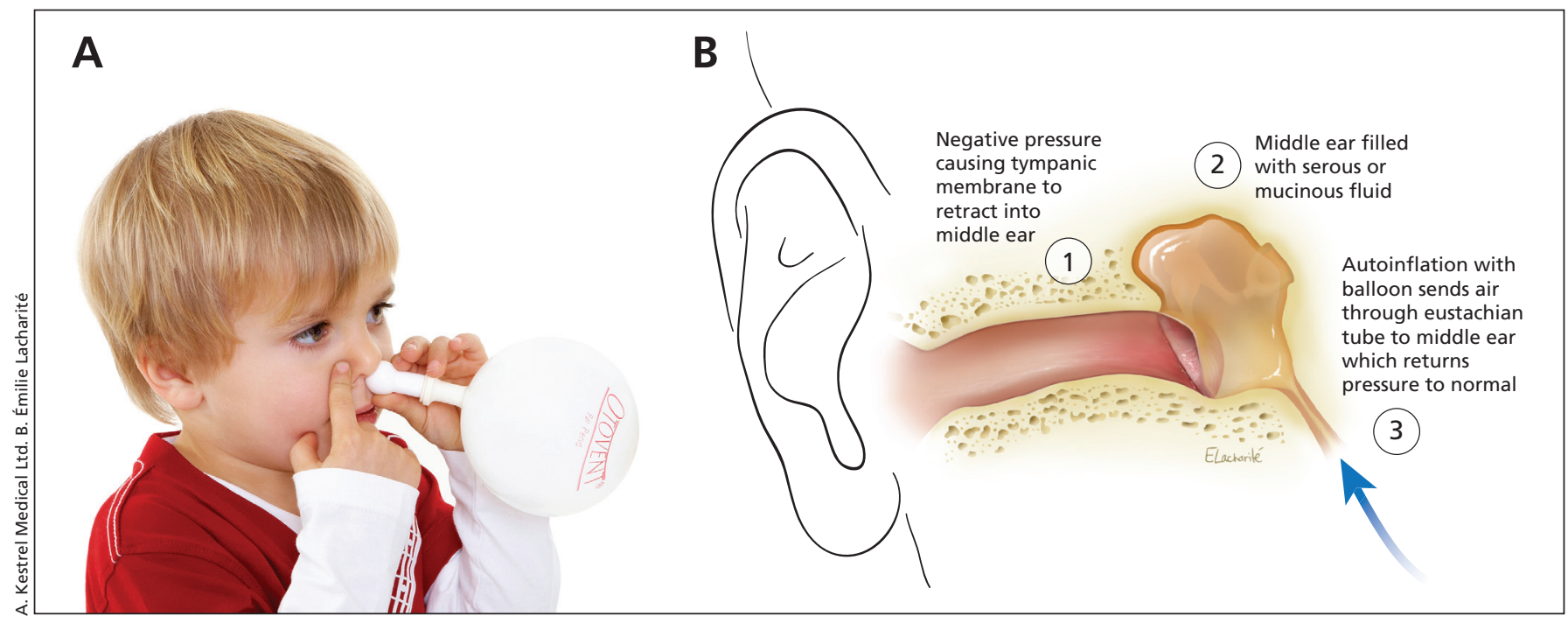

Figure 1: (A) Child demonstrating use of the Otovent device. Reproduced with permission. (B) Illustration of otitis media with effusion and the mechanism of autoinflation. 
surement could be made. The relative effect of autoinflation on the primary outcome at 1 month and at 3 months was estimated using a generalized linear model for binary data with log-link function, and adjusted for baseline covariates (tympanometric baseline severity, age, sex and primary care trust). We conducted an ear-based analysis at 1 and 3 months using generalized estimating equations.

We compared change from baseline quality of life (standardized OMQ-14 scores) using a linear mixed-effects model. Data from weekly symptom diaries of days with symptoms related to otitis media with effusion were summarized according to categories of the number of days with symptoms. Groups were compared using an ordinal logistic regression model.

Analyses were conducted using SAS version 9.3 and Stata version 13.0 according to a prespecified analysis plan.

\section{Results}

Between December 2011 and February 2013, 1235 children were screened for eligibility and $320(26 \%)$ were randomly assigned to standard care alone or autoinflation plus standard care (Figure 2). The main reasons for ineligibility

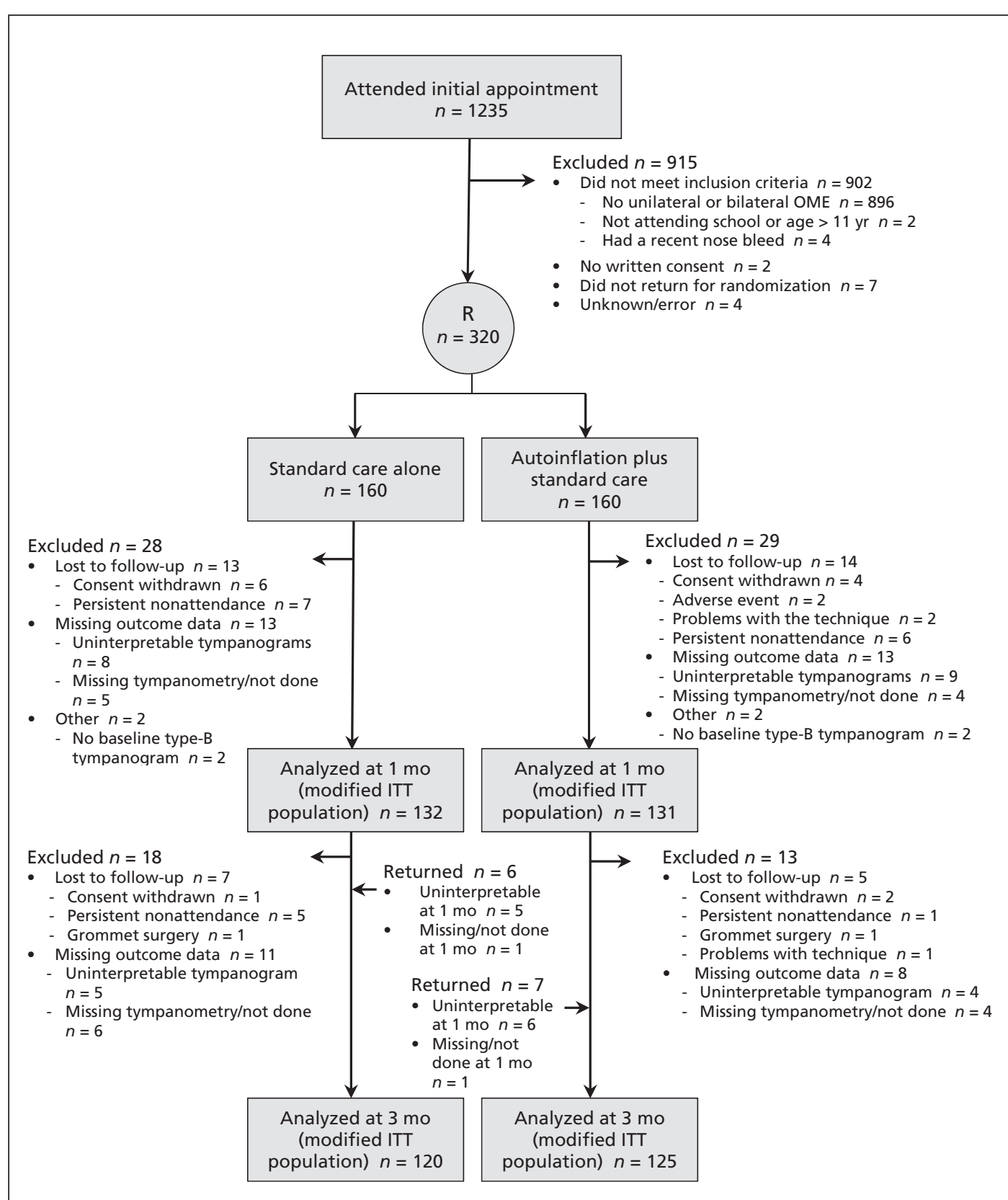

Figure 2: Enrolment, allocation and follow-up of patients. Note: ITT = intention-to-treat, OME = otitis media with effusion, $\mathrm{R}$ = randomization. 
Table 2: Baseline characteristics of participants, by treatment group

\begin{tabular}{|c|c|c|}
\hline \multirow[b]{2}{*}{ Characteristic } & \multicolumn{2}{|c|}{ No. \%* } \\
\hline & $\begin{array}{l}\text { Standard care } \\
\quad n=160\end{array}$ & $\begin{array}{l}\text { Autoinflation } \\
\quad n=160\end{array}$ \\
\hline Age, yr, mean \pm SD & $5.4 \pm 1.04$ & $5.4 \pm 1.24$ \\
\hline Male sex & $83(51.9)$ & $84(52.5)$ \\
\hline \multicolumn{3}{|l|}{ Severity of otitis media with effusion } \\
\hline No type-B tympanogram & $2(1.3)$ & $2(1.3)$ \\
\hline Type-B tympanogram, 1 ear & $91(56.9)$ & $90(56.3)$ \\
\hline Type-B tympanogram, 2 ears & 67 (41.9) & $68(42.5)$ \\
\hline \multicolumn{3}{|l|}{ Month randomly assigned } \\
\hline October to March & $107(66.9)$ & $107(66.9)$ \\
\hline April to September & $53(33.1)$ & $53(33.1)$ \\
\hline \multicolumn{3}{|l|}{ Ethnicity } \\
\hline White & $144(90.0)$ & $152(95.0)$ \\
\hline Bangladeshi/Indian & $2(1.3)$ & $2(1.3)$ \\
\hline Mixed race & $3 \quad(1.9)$ & $1 \quad(0.6)$ \\
\hline Other group & $2 \quad(1.3)$ & $2(1.3)$ \\
\hline No information & $(5.6)$ & $3 \quad(1.9)$ \\
\hline \multicolumn{3}{|l|}{ Education level of parent or caregiver } \\
\hline Attended school to age $16 \mathrm{yr}$; no certificate or diploma & $11 \quad(6.9)$ & $6 \quad(3.8)$ \\
\hline Attended school to age $16 \mathrm{yr}$; secondary school diploma & $28(17.5)$ & $33(20.6)$ \\
\hline College or nonuniversity certificate & $56(35.0)$ & $63(39.4)$ \\
\hline University degree & $37(23.1)$ & $31(19.4)$ \\
\hline Professional/postgraduate degree or certificate & $17(10.6)$ & $22(13.8)$ \\
\hline No information & $30(18.8)$ & $31(19.4)$ \\
\hline \multicolumn{3}{|l|}{ Parent-reported child characteristics } \\
\hline Asthmat & 19 (11.9) & $16(10.0)$ \\
\hline Eczema† & $15 \quad(9.5)$ & $20(12.5)$ \\
\hline Hay fevert & $40(25.0)$ & $42(26.3)$ \\
\hline Antibiotics in previous month $\dagger$ & 12 (7.5) & $21(13.1)$ \\
\hline $\begin{array}{l}\text { Parent-reported symptoms in the previous } 3 \text { mo } \\
\text { (children aged 4-6 yr only) }\end{array}$ & $n=135$ & $n=130$ \\
\hline A prolonged or bad cold, cough or chest infection & $113(83.7)$ & $119(91.5)$ \\
\hline Appears to be lip reading $\dagger$ & $27(20.0)$ & $27(20.8)$ \\
\hline An earache & $74(54.8)$ & $77(59.2)$ \\
\hline Not doing as well at school as expected $\dagger$ & $32(23.7)$ & $39(30.0)$ \\
\hline Often mishears what is said & $98(72.6)$ & $112(86.2)$ \\
\hline Has noises in the ear or is dizzy & $29(21.5)$ & $30(23.1)$ \\
\hline Hearing loss is suspected by anyone $\dagger$ & $56(41.5)$ & $67(51.5)$ \\
\hline Snores, blocked nose or poor sleep & $93(68.9)$ & $101(77.7)$ \\
\hline Says "eh what?" or "pardon" a lot & $107(79.3)$ & $114(87.7)$ \\
\hline Speech seems behind other children's & $22(16.3)$ & $31(23.8)$ \\
\hline Needs the television turned up & $78(57.8)$ & $82(63.1)$ \\
\hline Any suspected ear problem & $48(35.6)$ & $55(42.3)$ \\
\hline May be irritable or withdrawn & $43(31.9)$ & $38(29.2)$ \\
\hline No. of symptoms, median (IQR) & $6(4-8)$ & $7(5-9)$ \\
\hline OMQ-14 (quality of life)‡ & $n=153$ & $n=153$ \\
\hline Standardized score (SD) & $-0.04( \pm 0.95)$ & $0.07( \pm 1.00)$ \\
\hline \multicolumn{3}{|c|}{$\begin{array}{l}\text { Note: IQR = interquartile range, SD = standard deviation. } \\
\text { *Unless stated otherwise. } \\
\text { †Missing data for children in the standard care and autoinflation groups on the following variables: asthma and eczema }(n=9,4) \text {, } \\
\text { hay fever and antibiotics in previous month }(n=9,3) \text {, appears to be lip reading }(n=1 \text { in autoinflation), not doing as well at } \\
\text { school as expected and hearing loss is suspected by anyone }(n=1 \text { in standard care). } \\
\text { fLower scores represent better global ear-related health (range of scores }-2.1 \text { to 3.8). }\end{array}$} \\
\hline
\end{tabular}


were that children lacked a type-B tympanogram ( 2 children from each arm were subsequently withdrawn because of this), were not currently attending school or reported a recent nosebleed. Ineligible children reported fewer symptoms associated with otitis media with effusion in the preceding 3 months, and had fewer consultations for otitis media in the previous 12 months.

Baseline characteristics of children who were randomly assigned were balanced between the 2 groups (Table 2). Trial demographic data are comparable to national figures, but $33 \%$ of participating parents (v. $27 \%$ nationally) had a university or postgraduate degree. ${ }^{37}$

\section{Main results}

Retention was good, with $8.4 \%$ lost to follow-up at 1 month and $12.2 \%$ at 3 months. Uninterpretable tympanograms due to poor technique (leakage or low canal volume), and clinical problems (wax or perforation) were similar between groups, leaving 131 children in the autoinflation arm and 132 in the usual care arm in the modified intention-to-treat analysis.

Compared with standard care, children receiving autoinflation achieved tympanometric resolution more often at 1 month (adjusted relative risk [RR] 1.36, 95\% confidence interval [CI] 0.99 to 1.88 ) and at 3 months (adjusted RR $1.37,95 \%$ CI 1.03 to 1.83 , number needed to treat $[\mathrm{NNT}]=9)($ Table 3$)$. Sensitivity analyses using multiple imputation for missing data yielded similar but slightly smaller RRs that did not achieve statistical significance. Analyses for individual ears, adjusted for correlation between ears within child, showed that tympanometric resolution was significantly more likely with autoinflation at 1 month (adjusted RR $1.38,95 \%$ CI 1.01 to 1.87 ) and at 3 months (adjusted RR $1.41,95 \%$ CI 1.05 to 1.88 ), consistent with our per-child (primary) analysis (Table 3).

Table 3: Tympanometric resolution at 1 month and 3 months, by study group

\begin{tabular}{|c|c|c|c|}
\hline \multirow[b]{2}{*}{ Variable } & \multicolumn{2}{|c|}{ No. $(\%)$ of children* } & \multirow[b]{2}{*}{$\begin{array}{l}\text { Adjusted RR or OR }+ \\
(95 \% \mathrm{Cl})\end{array}$} \\
\hline & $\begin{array}{l}\text { Standard care } \\
\qquad n=160\end{array}$ & $\begin{array}{l}\text { Autoinflation } \\
\quad n=160\end{array}$ & \\
\hline 1-month analysis & $n=132$ & $n=131$ & \\
\hline \multirow{2}{*}{$\begin{array}{l}\text { Tympanometric resolution of } \geq 1 \text { type-B } \\
\text { ear per child at } 1 \text { moł }\end{array}$} & \multirow[t]{2}{*}{$47(35.6)$} & \multirow[t]{2}{*}{$62(47.3)$} & RR 1.36 (0.99-1.88)ף \\
\hline & & & RR $1.27(0.95-1.71) * *$ \\
\hline $\begin{array}{l}\text { Tympanometric resolution, by ear, } \\
\text { at } 1 \mathrm{mo}, \neq \S n=263 \text { children }\end{array}$ & $\begin{array}{c}n=187 \text { ears } \\
52(27.8)\end{array}$ & $\begin{array}{l}n=188 \text { ears } \\
73(38.8)\end{array}$ & RR 1.38 (1.01-1.87) \\
\hline Days with any symptom or problem & $n=138$ & $n=136$ & OR $0.66(0.41-1.05) \dagger \dagger$ \\
\hline None & $9(6.5)$ & $18(13.2)$ & \\
\hline $1-7$ & $47(34.1)$ & $49(36.0)$ & \\
\hline$\geq 8$ & $82(59.4)$ & $69(50.7)$ & \\
\hline 3-month analysis & $n=120$ & $n=125$ & \\
\hline \multirow{2}{*}{$\begin{array}{l}\text { Tympanometric resolution of } \geq 1 \text { type- } B \\
\text { ear at } 3 \text { moł }\end{array}$} & \multirow[t]{2}{*}{$46(38.3)$} & \multirow[t]{2}{*}{$62(49.6)$} & RR 1.37 (1.03-1.83)ף \\
\hline & & & RR $1.22(0.92-1.63) * *$ \\
\hline $\begin{array}{l}\text { Tympanometric resolution, } \\
\text { by ear, at } 3 \mathrm{mo}, \neq \S n=245 \text { children }\end{array}$ & $\begin{array}{c}n=166 \text { ears } \\
52(31.3)\end{array}$ & $\begin{array}{l}n=182 \text { ears } \\
74(40.6)\end{array}$ & RR $1.41(1.05-1.88)$ \\
\hline Days with any symptom or problem & $n=139$ & $n=139$ & OR $0.58(0.37-0.90)+\dagger$ \\
\hline None & $4(2.9)$ & $9(6.4)$ & \\
\hline $1-7$ & $29(20.9)$ & $30(21.6)$ & \\
\hline $8-28$ & $57(41.0)$ & $73(52.5)$ & \\
\hline$\geq 29$ & $49(35.3)$ & $27(19.4)$ & \\
\hline \multicolumn{4}{|c|}{$\begin{array}{l}\text { Note: } \mathrm{Cl}=\text { confidence interval, OR = odds ratio, RR = relative risk. } \\
\text { *Unless stated otherwise. } \\
\text { †ORs adjusted for age and sex. } \\
\text { †Adjusted for baseline severity ( } 1 \text { or } 2 \text { type-B ears), age and sex (not adjusted for centre effects due to nonconvergence). } \\
\text { §Generalized estimating equation model adjusting for correlation between ears for each child. } \\
\text { १Primary analysis: adjusted for baseline severity ( } 1 \text { or } 2 \text { type-B ear), age, sex and primary care trust. } \\
\text { **Sensitivity analysis: multiple imputation of all missing data using baseline variables (use of antibiotics, eczema, hay fever, } \\
\text { asthma, age, sex, baseline severity, baseline OMQ-14 and follow-up OMQ-14 weighted scores). } \\
\text { ††From ordinal logistic regression. }\end{array}$} \\
\hline
\end{tabular}




\section{Subgroup analyses}

We conducted prespecified subgroup analyses of effects of age ( $<6.5 \mathrm{yr} \mathrm{v}$. $\geq 6.5 \mathrm{yr}$ ), severity ( $1 \mathrm{v}$. 2 type-B ears at baseline), OMQ-14 standardized total score $(<0 \mathrm{v} . \geq 0)$ and sex on the primary outcome. In all cases we found no differences in treatment effects between subgroups. $P$ values for the interaction term (treatment by subgroup) in the model ranged from 0.3 to 0.5 (Appendix 2, available at www.cmaj.ca/lookup/suppl/ doi:10.1503/cmaj.141608/-/DC1).

\section{Ear-related quality of life and diary symptoms}

At 3 months, the mean change from baseline in the standardized OMQ-14 total scores was greater in the autoinflation arm than in the control arm by -0.33 points $(95 \% \mathrm{CI}-0.59$ to -0.07 ). The adjusted difference between groups was -0.42 points ( $95 \% \mathrm{CI}-0.63$ to -0.22 ) (Figure 3 ). This score difference represents a treatment effect size of 0.48 of an SD. The mean improvement in baseline score was -0.69 (0.84 SD) points at 3 months for the treatment arm. Effects were consistent across individual OMQ-14 items (Appendix 3, available at www.cmaj.ca/lookup/ suppl/doi:10.1503/cmaj.141608/-/DC1). Overall, children in the autoinflation arm had fewer days with any symptom or problem than children in the control arm at 1 month (median [interquartile range] 8 [2-16] v. 9 [4-17] d; OR 0.66, 95\% CI 0.41 to 1.05 ) and at 3 months (median [interquartile range] 14 [6-28] v. 22 [8-35] d; OR 0.58, $95 \%$ CI 0.37 to 0.90 ) (Table 3 ).

\section{Compliance}

A total of $89 \%$ of parents reported using the device "most" or "all of the time" during the first month of treatment, consistent with the daily compliance sticker charts. This level of compliance appears to be maintained in those continuing treatment up to 3 months (80\%).

\section{Adverse events}

We found very little difference between study arms in the number of children with nosebleed (15\% v. $14 \%)$, but there were more reported respiratory infections in the treatment group (15\% v. $10 \%$ of children). Most of the respiratory infections were mild afebrile rhinorrhea. Eight children receiving autoinflation (compared with 2 receiving usual care) reported otalgia (Table 4). Five children in the treatment group and 4 in the control group had an episode of acute otitis media. Two children in the treatment arm were withdrawn: 1 was admitted to hospital with mild/ early mastoiditis and made a full recovery, and a second was withdrawn due to otalgia.

\section{Interpretation}

In this study, we observed that autoinflation in young, school-aged children with otitis media with effusion is feasible in primary care and effective in clearing middle-ear effusions and improving symptoms and ear-related child and parent quality of life. Autoinflation is a simple, low-cost procedure that can be taught to young children in a primary care setting with a reasonable expectation of compliance. With an NNT of 9 , it is a relatively noninvasive option that can add benefit by helping to fill the current gap between either doing nothing effective or referring for surgery. ${ }^{13}$ Wider use of this device has considerable potential to address the present lack of treatment options for most symptomatic children, and the frequency with which inappropriate antibiotics continue to be used to fill this gap. ${ }^{9,12,13,21,24,25}$ Because fluid in the ear does not completely clear in many instances even after 3 months, and with a tendency to recur, clinical vigilance with the option for surgery remains crucial to evidence-based management.

Best evidence suggests there are currently no proven nonsurgical interventions for glue ear. Parents often see temporizing strategies as causing unreasonable delay, and this can also lead to use of ineffective treatments, such as antibiotics. The most recent Cochrane Review of autoinflation $^{28}$ highlighted the need for a large primary care study. The small hospital-based trials available $^{29-31}$ for meta-analysis did not assess autoinflation in a primary care setting and lack both power and generalizability to the majority of affected children. Adding our data (and unpublished pilot, $n=20$ ) to the meta-analysis more

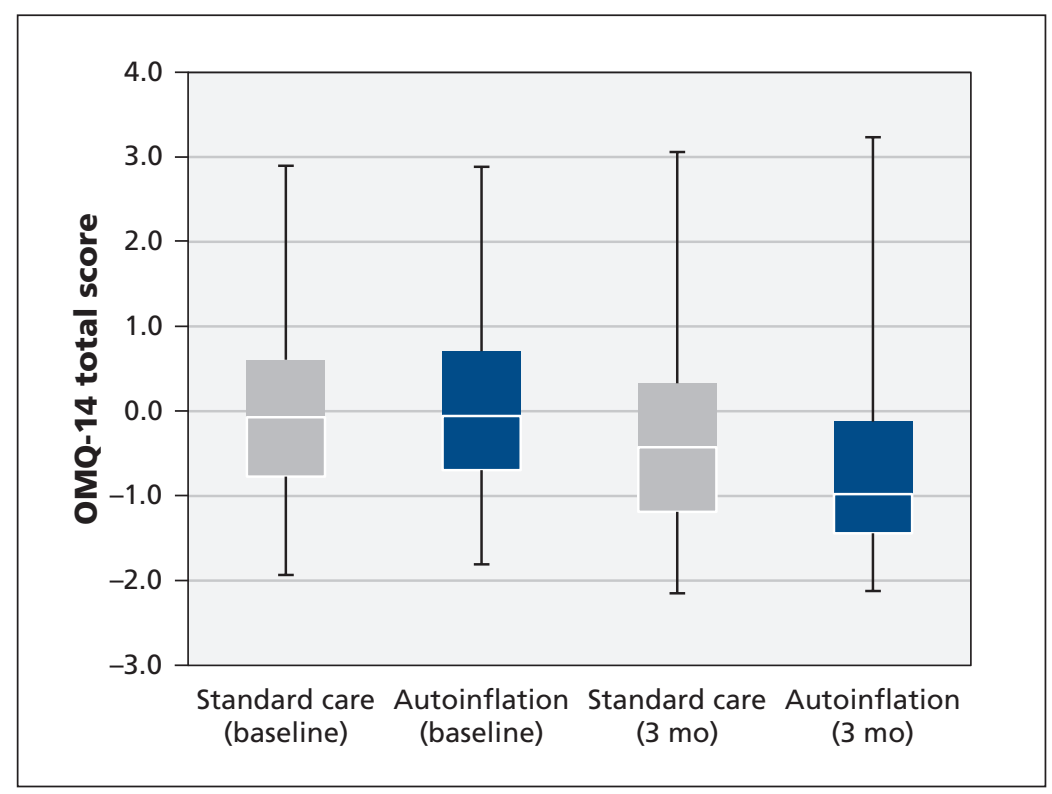

Figure 3: Standardized total scores on the OMQ-14 (an ear-related measure of quality of life) at baseline and at 3 months. Lower scores represent improvement. 
Table 4: Adverse events, by study group

\begin{tabular}{|c|c|c|c|c|}
\hline \multirow[b]{2}{*}{ Adverse event } & \multicolumn{2}{|c|}{$\begin{array}{c}\text { Standard care } \\
\quad n=160\end{array}$} & \multicolumn{2}{|c|}{$\begin{array}{l}\text { Autoinflation } \\
\quad n=160\end{array}$} \\
\hline & $\begin{array}{l}\text { No. of } \\
\text { events }\end{array}$ & $\begin{array}{l}\text { No. }(\%) \\
\text { affected } \\
\text { children }\end{array}$ & $\begin{array}{l}\text { No. of } \\
\text { events }\end{array}$ & $\begin{array}{l}\text { No. }(\%) \\
\text { affected } \\
\text { children }\end{array}$ \\
\hline Nosebleed & 26 & $24(15)$ & 26 & $22(14)$ \\
\hline Upper respiratory tract infection & 6 & $6 \quad(4)$ & 20 & 13 (8) \\
\hline Unspecified respiratory tract Infection & 4 & $4 \quad(3)$ & 9 & 9 \\
\hline Lower respiratory tract infection & 4 & $4 \quad(3)$ & 2 & 2 \\
\hline Acute otitis media & 4 & $4 \quad(3)$ & 6 & 5 \\
\hline Otalgia & 2 & $2(1)$ & 8 & (4) \\
\hline Headache & - & - & 2 & 2 \\
\hline Hay fever & - & - & 1 & (1) \\
\hline Hospital admission* & 1 & 1 (1) & 1 & 1 \\
\hline
\end{tabular}

than doubles the available sample size with an estimated aggregate effect size of 1.61 (95\% CI 1.26 to $2.06, I^{2}$ heterogeneity $0.0 \%$ ) (Appendix 4, available at www.cmaj.ca/lookup/suppl/ doi:10.1503/cmaj.141608/-/DC1).

For the child, parent and professional, the main issue is the impact caused by otitis media with effusion. ${ }^{38,39}$ From this consequential perspective, moderate improvement in the total OMQ-14 score is important and encouraging (e.g., in terms of reduced days with hearing difficulty over $3 \mathrm{mo}$ ) (Appendix 3). Published data on ear-related quality of life from other trials of otitis media with effusion are currently very sparse. ${ }^{9,15,26}$

\section{Limitations}

The main limitation of this study is that the intervention cannot be blinded, and Hawthorne effects are possible. ${ }^{40}$ However, concealment issues are unlikely to affect tympanometric outcomes, because Web-based randomization was used for allocation, and all printouts were anonymized and assessed by experts who were unaware of allocation. Even if symptom and mapped quality of life (OMQ-14) scores were affected by performance bias, the effects observed would still be likely to reflect routine practice. The study population included children who were likely to be able to reliably perform autoinflation (i.e., age $\geq 4 \mathrm{yr}$ ). Although children of all ages frequently present to primary care, in the UK the most common age for referral for otitis media with effusion is between 3.5 and 8 years. ${ }^{7,9,26}$ This study does not address treatment for the youngest group of children affected.
However, children as young as 3 years have been found to be able to use the device in hospital settings. ${ }^{29,31}$

\section{Conclusion}

We have found use of autoinflation in young, school-aged children with otitis media with effusion to be feasible, safe and effective in clearing effusions, and in improving important ear symptoms, concerns and related quality of life over a 3-month watch-and-wait period. Autoinflation may not be suitable for all children, especially those under 4 years of age, and does require ongoing commitment to treatment. Further research is needed for very young children, and to inform prudent use across different health settings. The method has scope to be used in many symptomatic children, and is capable of producing better management and outcomes in primary health care systems. Wider use of nasal balloon autoinflation could address the present lack of treatment options for children with symptomatic otitis media with effusion.

\section{References}

1. Zielhuis GA, Rach GH, van den Broek P. Screening for otitis media with effusion in preschool children. Lancet 1989;1:311-4.

2. Stool SEBA, Berman S, Carney CJ, et al. Otitis media with effusion in children. Rockville (MD): Agency for Healthcare Research and Quality; 2013.

3. Fry J, Sandler G. Common diseases. 5th ed. New York: Kluwer Academic Publishers; 1993.

4. Williamson IG, Dunleavey J, Bain J, et al. The natural history of otitis media with effusion - a three-year study of the incidence and prevalence of abnormal tympanograms in four South West Hampshire infant and first schools. J Laryngol Otol 1994;108: 930-4.

5. Higson J, Haggard M. Parent versus professional views of the developmental impact of a multi-faceted condition at school age: otitis media with effusion ('glue ear'). Br J Educ Psychol 2005;75:623-43. 
6. Maw AR. Glue ear in childhood. London (UK): Mac Keith Press; 1995.

7. Browning GG. Two-year outcome of ventilation tubes in a randomized controlled trial of persistent childhood otitis media with effusion. Clin Otolaryngol Allied Sci 2001;26:342-4.

8. Browning GG. Watchful waiting in childhood otitis media with effusion. Clin Otolaryngol Allied Sci 2001;26:263-4.

9. Williamson I, Benge S, Barton S, et al. Topical intranasal corticosteroids in 4-11 year old children with persistent bilateral otitis media with effusion in primary care: double blind randomised placebo controlled trial. BMJ 2009;339:b4984.

10. van Balen FA, de Melker RA, Touw-Otten FW. Double-blind randomised trial of co-amoxiclav versus placebo for persistent otitis media with effusion in general practice. Lancet 1996; 348:713-6.

11. Casselbrant ML, Brostoff LM, Cantekin EI, et al. Otitis media with effusion in preschool children. Laryngoscope 1985;95:428-36.

12. Williamson I, Benge S, Mullee M, et al. Consultations for middle ear disease, antibiotic prescribing and risk factors for reattendance: a case-linked cohort study. Br J Gen Pract 2006;56:170-5.

13. Surgical management of otitis media with effusion in children. London (UK): National Collaborating Centre for Women's and Children's Health; 2008.

14. Marchisio P, Grasso D. Using differences and similarities between centres for power and generality. Helsinki (Finland): ESPO Conference; May 27, 2010.

15. Dakin H, Petrou S, Haggard M, et al. Mapping analyses to estimate health utilities based on responses to the OM8-30 Otitis Media Questionnaire. Qual Life Res 2010;19:65-80.

16. American Academy of Family Physicians; American Academy of Otolaryngology-Head and Neck Surgery; American Academy of Pediatrics Subcommittee on Otitis Media With Effusion. Otitis media with effusion. Pediatrics 2004;113:1412-29.

17. Cullen KA, Hall MJ, Golosinskiy A. Ambulatory surgery in the United States 2006. Hyattsville (MD): National Center for Health Statistics; 2009.

18. Desai SN, Kellner JD, Drummond D. Population-based, agespecific myringotomy with tympanostomy tube insertion rates in Calgary, Canada. Pediatr Infect Dis J 2002;21:348-50.

19. van Dongen TM, van der Heijden GJ, Freling HG, et al. Parentreported otorrhea in children with tympanostomy tubes: incidence and predictors. PLOS ONE 2013;8:e69062.

20. Grommet insertion and adenoidectomy for otitis media with effusion. Dublin (Ireland): Health Information and Quality Authority; 2013.

21. van Zon A, van der Heijden GJ, van Dongen TM, et al. Antibiotics for otitis media with effusion in children. Cochrane Database Syst Rev 2012;9:CD009163.

22. Simpson SA, Lewis R, van der Voort J, et al. Oral or topical nasal steroids for hearing loss associated with otitis media with effusion in children. Cochrane Database Syst Rev 2011;CD001935.

23. Griffin G, Flynn CA. Antihistamines and/or decongestants for otitis media with effusion (OME) in children. Cochrane Database Syst Rev 2011;CD003423.

24. Davies S, Grant J, Catchpole M. The drugs don't work: a global threat. New York: Penguin; 2013.

25. Antimicrobial resistance: global report on surveillance. Geneva (Switzerland): World Health Organization; 2014. Available: www. who.int/drugresistance/documents/surveillancereport/en (accessed 2014 May 6).

26. Browning GG, Rovers MM, Williamson I, et al. Grommets (ventilation tubes) for hearing loss associated with otitis media with effusion in children. Cochrane Database Syst Rev 2010;CD001801.

27. Williamson I. Otitis media with effusion. BMJ Clin Evidence Jan. 12, 2011 [Epub ahead of print]. Available: www.clinicalevidence. com/x/systematic-review/0502/overview.html (accessed 2014 May 6).

28. Perera R, Glasziou PP, Heneghan CJ, et al. Autoinflation for hearing loss associated with otitis media with effusion. Cochrane Database Syst Rev 2013;5:CD006285.

29. Blanshard JD, Maw AR, Bawden R. Conservative treatment of otitis media with effusion by autoinflation of the middle ear. Clin Otolaryngol Allied Sci 1993;18:188-92.

30. Ercan I, Cakir B, Kayaoglu S, et al. Long term effect of autoinflation in the treatment of otitis media with effusion. $K B B$ Forum 2005;4:166-70.

31. Stangerup SE, Sederberg-Olsen J, Balle V. Autoinflation as a treatment of secretory otitis media. A randomized controlled study. Arch Otolaryngol Head Neck Surg 1992;118:149-52.

32. Jerger J. Clinical experience with impedance audiometry. Arch Otolaryngol 1970;92:311-24

33. Fiellau-Nikolajsen M. Epidemiology of secretory otitis media A descriptive cohort study. Ann Otol Rhinol Laryngol 1983;92: $172-7$
34. Cantekin EI, Bluestone CD, Fria TJ, et al. Identification of otitis media with effusion in children. Ann Otol Rhinol Laryngol Suppl 1980;89:190-5.

35. Browning GC. Paediatric otolaryngolgy. Chapter 72. In: ScottBrown's otorhinolaryngology, head and neck surgery. Vol 1, pt 12. 7th ed. London (UK): Hodder Arnold; 2008.

36. Feeny D, Furlong W, Torrance GW, et al. Multiattribute and single-attribute utility functions for the health utilities index mark 3 system. Med Care 2002;40:113-28.

37. Qualifications and students, local authorities in the United Kingdom. London (UK): Office for National Statistics; 2011. Available: www.ons.gov.uk (accessed 2014 Jan. 8).

38. Rosenfeld RM, Goldsmith AJ, Tetlus L, et al. Quality of life for children with otitis media. Arch Otolaryngol Head Neck Surg 1997; 123:1049-54.

39. Hearing and balance disorders. Achieving excellence in diagnosis and management. London (UK): Royal College of Physicians; 2007.

40. McCarney R, Warner J, Iliffe S, et al. The Hawthorne effect: a randomised, controlled trial. BMC Med Res Methodol 2007;7:30.

Affiliations: Primary Care and Population Sciences (Williamson, Vennik, Little), Faculty of Medicine, University of Southampton, Aldermoor Close, Southampton, UK; Nuffield Department of Primary Care Health Sciences (Harnden, Voysey, Perera, Kelly, Mant), University of Oxford, Oxford, UK; Faculty of Medicine (Yao, Raftery), University of Southampton, Southampton General Hospital, Southampton, UK

Contributors: Ian Williamson conceived and designed the study, led protocol development, funding application, analysis and interpretation. Jane Vennik provided day-to-day management, coordinated recruitment, and contributed to the data collection, analysis and interpretation. Anthony Harnden contributed to protocol development, funding application and data interpretation. Merryn Voysey led the statistical analysis and interpretation of the study findings. Rafael Perera contributed to protocol development, funding application, statistical analysis and data interpretation. Sadie Kelly supervised the randomization process, and coordinated data collection, cleaning and validation. Guiqing Yao and James Raftery led the health economic analysis. David Mant contributed to protocol development, funding application and interpretation of findings. Paul Little contributed to protocol development, funding application and interpretation of findings. All of the authors contributed to drafting the article. Ian Williamson and Jane Vennik revised the arti$\mathrm{cle}$, with particular support from Merryn Voysey and Anthony Harnden. All of the authors gave final approval of the version to be published and agreed to act as guarantors of the work.

Funding: This project was funded by the Health Technology Assessment program of the National Institute for Health Research and will be published in full in Health Technology Assessment (www.nets.nihr.ac.uk). The funders had no role in the design or conduct of the study, the analysis or interpretation of the results, or the decision to submit the manuscript for publication.

Acknowledgements: The authors thank the following: the Health Technology Assessment and National Institute for Health Research funders; the Oxford Primary Care Clinical Trials Unit, especially Sarah Benge for protocol development, logistical help and pilot work; Sharon Charnock of Starkey Laboratories Ltd. for audiological review and assistance with nurse training; Mark Haggard and Helen Spencer for use of the OMQ-14, with advice and supply of a scoring system; and Sven-Eric Stangerup, the inventor of Otovent, for advice on use. The authors also thank the regional primary care research networks and all participating practices and Kestrel Medical Ltd. for the trial supply of Otovent and providing the photograph in Figure 1. Finally, and most importantly, the authors thank all the children and families who took part in the study. See the following video online: Demonstration of nasal balloon autoinflation. www.cmaj.ca/lookup/ suppl/doi:10.1503/cmaj.141608/-/DC2 